\title{
Demographic characteristics of patients undergoing aortic valve replacement for stenosis: relation to valve morphology
}

\author{
M J Davies, T Treasure, D J Parker
}

\begin{abstract}
Objective-To determine the relative importance of the different causes of isolated aortic valve stenosis in a surgical series, and to relate these to patient characteristics including the rate of insertion of bypass grafts for coronary artery disease.
\end{abstract}

Design-Survey of the clinical and pathological data on patients undergoing aortic valve replacement for isolated stenosis.

Setting-Tertiary care cardiothoracic surgical unit.

Patients and methods-465 adult patients undergoing aortic valve replacement representing a consecutive series in one surgical unit. Retrospective review of patients records and classification of cause of aortic stenosis based on pathological examination of excised valve cusps.

Results $-63 \cdot 7 \%$ patients had calcific bicuspid valves, $26.9 \%$ tricuspid calcific valves, and $5 \cdot 4 \%$ rheumatic, $2 \cdot 6 \%$ mixed pathology and $1.5 \%$ unicommissural valves. The ratio of males to females for bicuspid valves was $1 \cdot 85: 1$ and for tricuspid calcific valves $0 \cdot 76: 1$. The mean age of patients with bicuspid valves was 64.9 years compared with 73.4 years for those with tricuspid valves. Some $22.3 \%$ of patients with bicuspid valves and $44.8 \%$ of those with tricuspid valves had sufficient coronary artery disease to necessitate insertion of coronary bypass grafts. The differential rate of insertion of coronary bypass grafts was independent of age.

Conclusions-Bicuspid calcified aortic valves are the predominant cause of isolated aortic valve stenosis followed by tricuspid calcified aortic valves. The sex and age distribution of bicuspid and tricuspid calcific aortic valve stenosis is different. The higher rate of insertion of vascular grafts in tricuspid calcific aortic valves may indicate that risk factors for atherosclerosis enhance cusp calcification in these patients.

(Heart 1996;75:174-178)

Keywords: aortic valve; stenosis; calcification; atheroslcerosis

Before 1950 rheumatic disease was considered to be the predominant cause of isolated aortic valve stenosis. Morphological studies, however, suggested that many stenotic aortic valves were bicuspid and had undergone dystrophic calcification in the absence of the pathological features of chronic rheumatic disease. ${ }^{1-3} \mathrm{~A}$ range of well defined entities causing aortic valve stenosis have now been characterised by their morphological features, and can be recognised in situ at the time of surgery and in the excised cusp tissue. ${ }^{4-6}$ These entities include bicuspid and tricuspid aortic valves in which cusp calcification causes obstruction and commissural fusion is absent. Rheumatic disease $^{7}$ is characterised by fusion of the three commissures to produce a small triangular valve orifice and can be readily distinguished from bicuspid calcific valves. In addition to these causes, there are small numbers of cases in which one commissure only is present producing an elliptical peripherally sited orifice and bicuspid valves in which there is commissural fusion in addition to cusp calcification. The present study examines the characteristics of patients with these different morphological forms of aortic valve stenosis.

\section{Patients and methods}

The study comprised 465 patients who had aortic valve replacement for dominant aortic stenosis and in whom the cause of stenosis could be ascertained. These represent a consecutive series of patients over a 10 year period. Fifty six patients were excluded from the study because the cause could not be ascertained. In such cases piecemeal surgical removal of the valve often precluded a cause being ascertained or the valve was not submitted for pathological examination. Patients in whom concomitant mitral valve surgery was required were not included. Valves were characterised by the number of cusps, distribution of calcification, and the presence or absence of commissural fusion (fig 1). The distinction between a rudimentary commissure formed before birth and acquired fusion of two adjacent cusps later in adult life was made by the presence or absence of the residual free edges of the cusps extending to the aortic wall. In bicuspid valves the anatomy as recorded at surgery was used to determine the relation of cusp arrangement to the coronary orifices. The number of saphenous vein and internal mammary grafts inserted was taken from the surgical operation records. The relation among age, gender, valve morphology, and whether coronary bypass grafts were inserted was studied by multiple logistic regression. 
Figure 1 (A) Bicuspid aortic valve stenosis. There are two cusps without commissural fusion. One cusp (arrows) contains a large nodular mass of calcium. (B) Tricuspid calcific aortic stenosis. There are three cusps without commissural fusion. All the cusps contain symmetrically distributed nodular masses of calcification (arrows). (C) Rheumatic aortic stenosis. There is even fusion of the three commissures (arrows) to produce a central triangular orifice. (D) Mixed rheumatic/bicuspid aortic stenosis. The valve is bicuspid with calcification in both cusps. One commissure is fused (arrows). (E)

Unicommissural aortic stenosis. The valve has an elliptical eccentric orifice. Raphès mark rudimentary commissures (arrows).
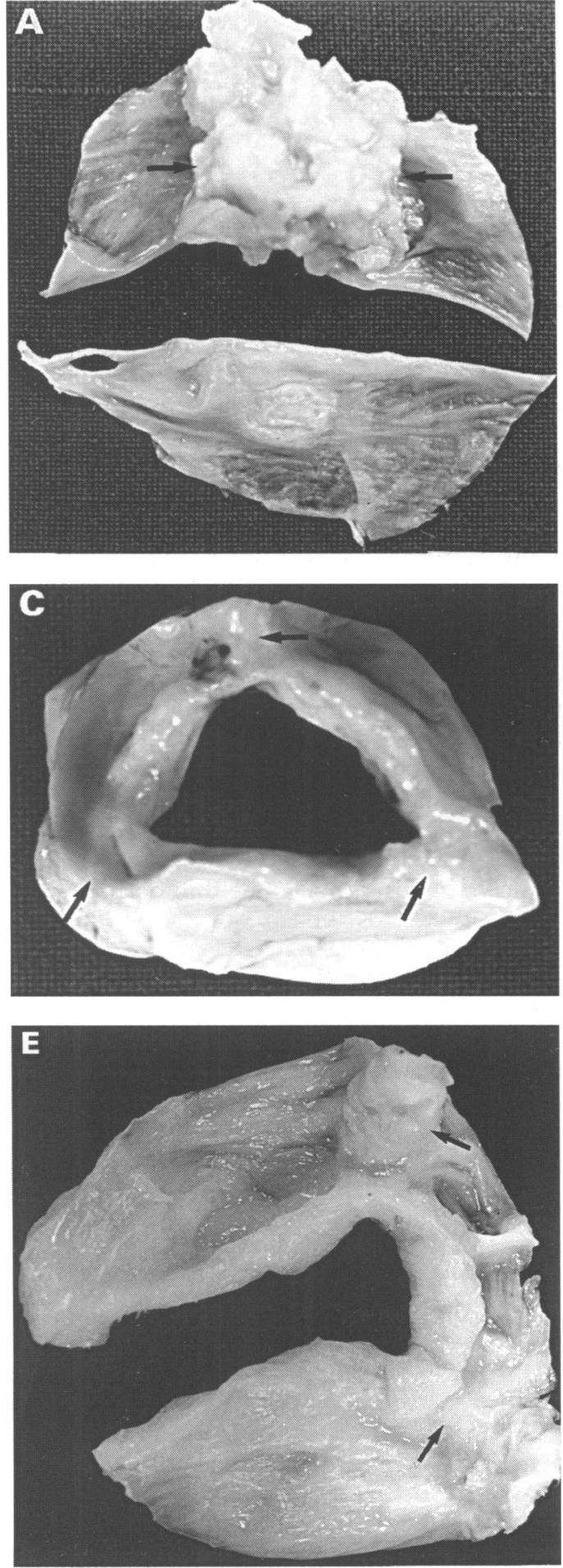

\section{Results}

Table 1 gives the valve pathology in 465 patients. Calcification in bicuspid valves was variable in degree and distribution. When calcification was confined to one cusp (fig 1) it was maximal in the raphè area. Many bicuspid

Table 1 Pathological causes of aortic valve stenosis

\begin{tabular}{lc}
\hline Valve pathology & No (\%) \\
\hline Bicuspid calcific & $296(63 \cdot 7)$ \\
Bicuspid calcific with commissural fusion & $12(2 \cdot 6)$ \\
Unicommisural & $7(1 \cdot 5)$ \\
Rheumatic & $25(5 \cdot 4)$ \\
Tricuspid calcific & $125(26 \cdot 9)$ \\
Total & 465 \\
\hline
\end{tabular}
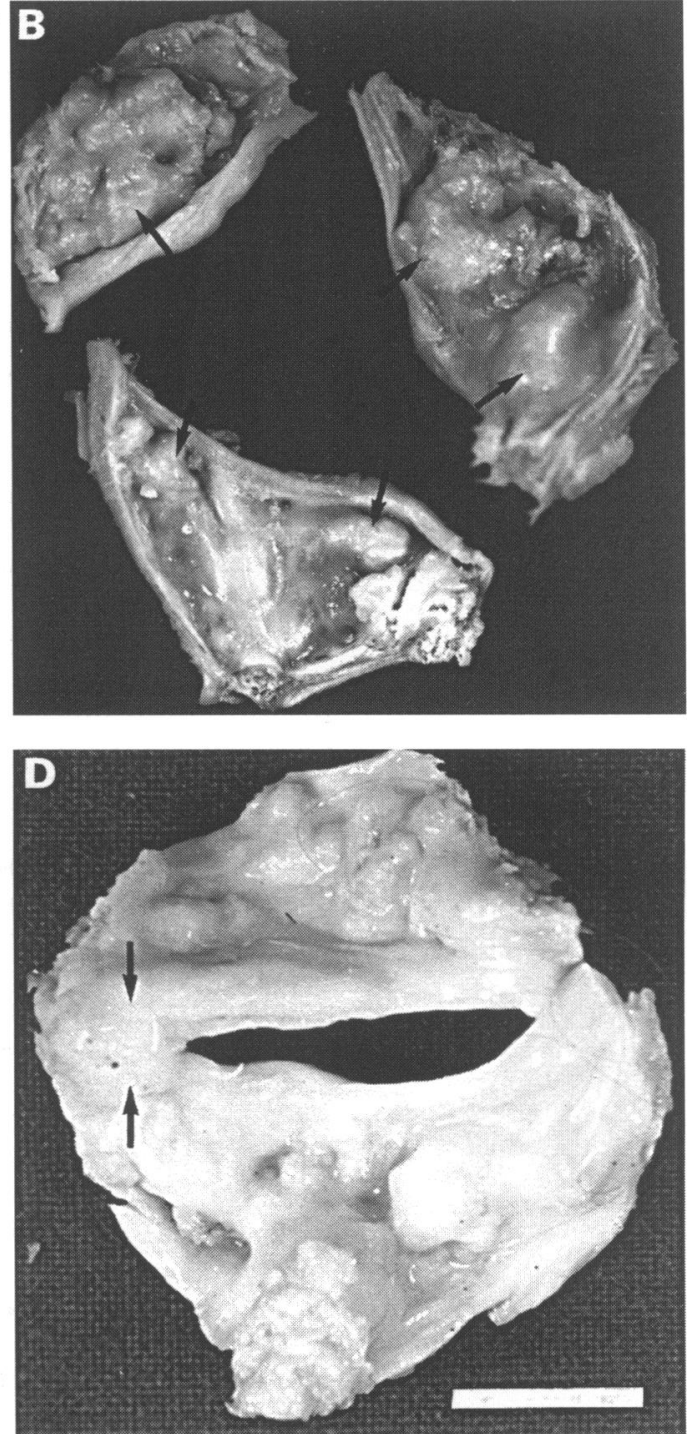

valves, however, had developed nodular masses of calcification in both cusps. Calcification in tricuspid valves was symmetric with nodules and bars of calcium distributed along the bases of the cusps at the line of max-

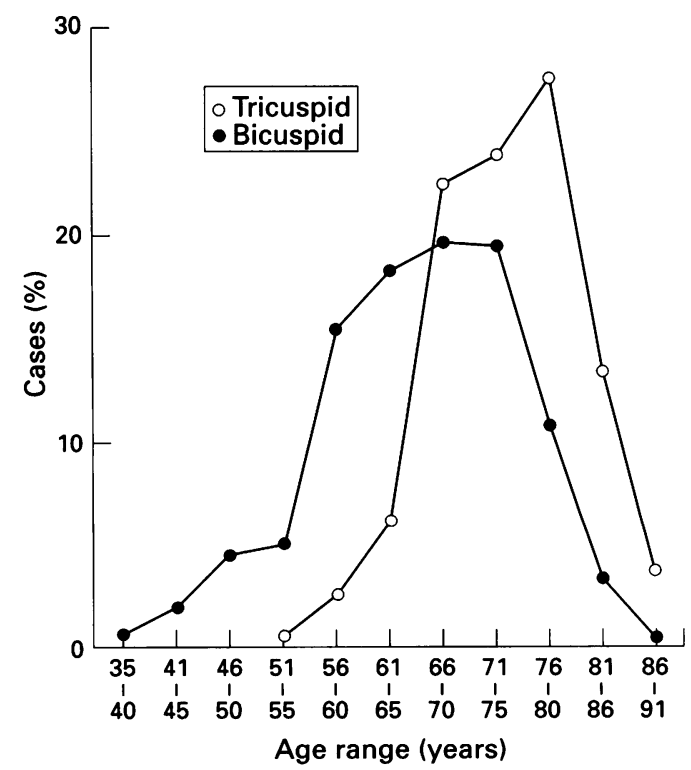

Figure 2 Age distribution of bicuspid and tricuspid aortic valve stenosis. 
Table 2 Rates of insertion of bypass grafts by age and valve type

\begin{tabular}{lllll}
\hline & \multicolumn{5}{l}{ Bypass grafts (\%) by age (years) } \\
\cline { 2 - 5 } Valve & $<60$ & $61-70$ & $71-80$ & $>81$ \\
\hline Bicuspid & $15 \cdot 8$ & $23 \cdot 6$ & $24 \cdot 8$ & $16 \cdot 7$ \\
Tricuspid & 40.0 & $53 \cdot 4$ & $44 \cdot 1$ & $36 \cdot 4$ \\
\hline
\end{tabular}

Table 3 Multiple logistic regression of the affect of valve type, gender and age on the need to insert coronary bypass grafts

\begin{tabular}{lccccc}
\hline Variable & $d f$ & $\begin{array}{l}\text { Parameter } \\
\text { estimates }\end{array}$ & $S E$ & $P^{*}$ & $\begin{array}{l}\text { Odds } \\
\text { ratio }\end{array}$ \\
\hline Intercept & 1 & 3.88 & 0.85 & 0.001 & 48.50 \\
Valve type & 1 & -1.12 & 0.26 & 0.001 & 0.33 \\
Age & 1 & -0.01 & 0.02 & 0.2734 & 0.99 \\
Male gender & 1 & 0.45 & 0.24 & 0.0607 & 1.57 \\
\hline${ }^{*} \chi^{2}$ test. & & & &
\end{tabular}

imal flexion. In tricuspid and bicuspid valves the calcium nodules projected into the sinuses. Six $(24 \%)$ of the 25 rheumatic valves showed nodular calcification maximal at the fused commissures.

The gender distribution of tricuspid and bicuspid calcific stenosis differs. The ratio of males to females for bicuspid calcific stenosis is $1 \cdot 85: 1$ and that for tricuspid calcific stenosis $0 \cdot 76: 1$. The mean (range) age for bicuspid calcific stenosis was 64.9 (31-93) years at operation and that for tricuspid calcific stenosis 73.4 (55-95) years (fig 2). Sixty six (22.3\%) of 296 patients with bicuspid valves had coronary bypass grafts, while $56(44 \cdot 8 \%)$ of 125 with tricuspid aortic valves had bypass grafts
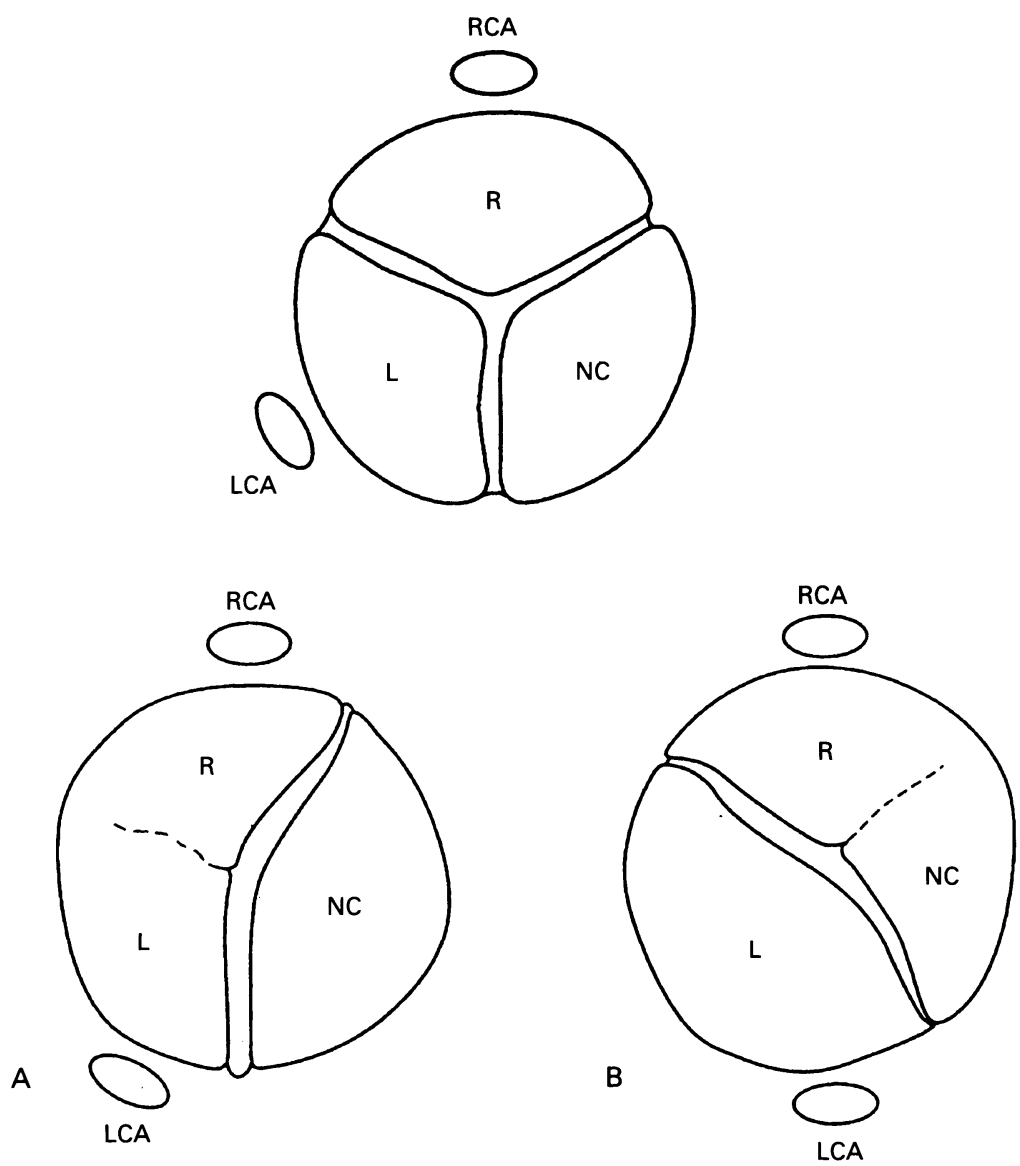

Figure 3 Arrangement of cusps to coronary orifices in bicuspid valves. (A) Both arteries open from behind one cusp. (B) $A$ coronary orifice lies behind each cusp. RCA, right open from behind one cusp. (B) A coronary
coronary artery; $L C A$, left coronary artery. inserted. The mean number of grafts in each group was identical ( 1.8 per patient). Differential rates of insertion of vein grafts between tricuspid and bicuspid calcific valves existed at all ages (table 2). Multiple logistic regression (table 3 ) showed that valve type had the greatest influence on the odds of a coronary bypass graft being needed with a significantly different odds ratio of 0.33 where a value of 1 would indicate no influence of valve type. Age did not influence the differential rate, but there was a trend to higher rates of insertion of grafts in males in both types of valve $(P>0.06)$

In 94 patients with bicuspid aortic valves in which the coronary artery anatomy was recorded in detail at operation, $13(13.8 \%)$ had a coronary artery arising behind each cusp and $81(86.2 \%)$ had both arteries arising from behind one of the two cusps (fig 3).

The mean (range) age of 25 patients with rheumatic-type valve morphology was $62 \cdot 7$ (34-81) years. The mitral valve was noted to be abnormal but was not replaced in eight patients. The mean (range) age of patients with unicommissural stenosis was $45 \cdot 6$ (23-63) years. In one of the 12 patients in whom there was a bicuspid valve with superadded commissural fusion the mitral valve was noted to be abnormal at operation but did not require surgical intervention.

\section{Discussion}

Any surgical series is biased by the age and geographical population from which it is drawn. The present series was from a population in which acute rheumatic fever had virtually vanished by 1950 and the number of patients with chronic rheumatic aortic stenosis was correspondingly low. Series from Western countries showed about one third of cases to be due to chronic rheumatic disease up to 1975 with a steady decline thereafter to between 5 and $15 \%$. The proportion of cases due to rheumatic disease in the present study is too small for useful analysis but does show the increased age of such patients compared with that in other populations in which acute rheumatic disease is still common or in series reported from the developed world before 1970. The addition of commissural fusion to a bicuspid calcified valve has been used to suggest previous episodes of rheumatic fever arguing that patients with bicuspid valves are unlikely to be spared streptococcal infection in youth. ${ }^{8}$ In the present study, however, other markers of rheumatic disease, such as an abnormal mitral valve were rare, and acquired commissural fusion may simply be the secondary result of abnormal flow through a greatly disorganised valve.

The two major causes of aortic valve stenosis in most geographical populations are now bicuspid and tricuspid calcific aortic stenosis. The age distribution of bicuspid calcific stenosis has been emphasised in several reports ${ }^{2910}$ and essentially it presents in late middle age. Individual cases, however, may continue to present into old age. ${ }^{11}$ As the frequency of a 
bicuspid valve is estimated to be as high as $1 \%$ in the general population ${ }^{12}$ not all can ultimately become sufficiently calcified to produce obstruction.

Tricuspid calcific aortic valve stenosis has a different age distribution reflected in sobriquet senile aortic stenosis. The increased longevity of life in Western populations coupled with greater use of cardiac surgery in the elderly has produced an apparent increase in the condition in recent years. ${ }^{10}$ There is good biochemical evidence that the calcium content of normal aortic valves rises steadily with age, a process that accelerates over the age of $50 .{ }^{13}$ Nevertheless, echocardiographic studies do not suggest that if everyone lived long enough they would develop aortic stenosis. The prevalence of aortic valve calcification in a random elderly population was low. ${ }^{14}$ The present series had sufficient patients to contrast bicuspid and tricuspid calcific aortic stenosis. The former is male predominant and the latter female. Although the age ranges mostly overlapped, on average patients with bicuspid stenosis were a decade younger than those with tricuspid stenosis.

There are three views on the pathogenesis of cusp calcification in bicuspid and tricuspid aortic valves. Calcification may be an agerelated mechanical wear and tear phenomenon. The earlier calcification of bicuspid valves relates to greater mechanical stress on the abnormal cusps. The more abnormal bicuspid valves with a raphè and unequal sized cusps may be those at greater risk of calcification. Calcification in bicuspid valves develops within the fibrous core of the cusp ${ }^{15}$ before bulging towards the aortic aspect of the cusp facing the sinus.

A similar mechanism may cause calcification in tricuspid valves. Calcium is deposited evenly in each of the three cusps in a $C$ shaped mass at the sites of cusp flexion and such a distribution is in accord with areas of greatest mechanical stress. Even in normal aortic valves there is a considerable variation in the areas of the three cusps ${ }^{16}$ and it could be that those with greatest variation are at greatest risk of calcification.

A different mechanism, however, has been proposed for calcification of tricuspid aortic valves. The process has been postulated to begin on the aortic face of the cusps not within the core, and calcified nodules project into the sinus rather than extending deep into the substance of the cusp. ${ }^{15}$ Calcification is also associated with insudation of lipid and some accumulation of macrophages. ${ }^{17}$ Calcification promoting factors such as osteopontin have been demonstrated in such macrophage infiltrates. The process of calcification in tricuspid valves has therefore been likened to atherosclerosis and a role for plasma lipids postulated.

The evidence for linking hyperlipidaemia to an increased risk of aortic valve calcification is, however, conflicting. A comparison of 71 patients with tricuspid calcific aortic stenosis with 109 patients with bicuspid calcific aortic stenosis showed that a high body mass index, a serum total cholesterol of over $200 \mathrm{mg} / \mathrm{dl}(5 \cdot 2$ $\mathrm{mmol} / \mathrm{l}$ ) and the presence of concomitant coronary disease were associated with tricuspid valves in a logistic regression model..$^{18}$ In contrast, another study of 39 patients with bicuspid valves and 30 with tricuspid valves found gender and smoking to be risk factors selecting for tricuspid calcific stenosis. ${ }^{19}$

Another study using echocardiography to detect non-stenotic aortic valve calcification in 501 patients aged from 55 to 86 years found no correlation with lipid levels or smoking but there were highly significant correlations with hypertension and low body mass; no link with male gender was found. ${ }^{20}$

The most compelling evidence linking tricuspid calcific stenosis with atherosclerosis is the frequency of concomitant coronary disease. In the present study the rate of insertion of coronary bypass grafts was doubled in tricuspid valves compared with that in bicuspid valves and this was independent of age. It may be, however, that the presence of coronary disease, in addition to aortic valve disease in elderly patients, selects specifically for referral for surgery thus overestimating the degree of associated coronary disease. We do not have reliable data on the lipid levels of the patients or their smoking histories because this was a retrospective study going back over 10 years.

A third aspect of valve calcification is the individual propensity to calcify extra-skeletal tissues. Paget's disease of bone, ${ }^{21}$ chronic renal failure, ${ }^{22}$ and primary hyperparathyroidism ${ }^{23}$ are known to produce calcific tricuspid aortic valve stenosis. This had led to the view that the serum calcium phosphorus ratio determines the risk of calcifying the aortic valve in elderly patients..$^{20}$ It has been shown that there is an association between reduced bone mineral density and aortic valve calcification. ${ }^{25}$ Excess mobilisation of bone calcium in osteoporosis may be a factor in the female preponderance of senile calcific aortic stenosis.

Cusp calcification is therefore caused by the interplay of three pathogenetic mechanisms: mechanical wear and tear which is more important in bicuspid aortic valves; a tendency for the individual to calcify extra-skeletal tissues; and thirdly, risk factors for atherosclerosis leading to calcification of the aortic cusps. In the present study the need for insertion of coronary artery grafts was higher in patients with tricuspid calcific aortic stenosis than in those with a bicuspid calcified valve. This suggests that risk factors for atherosclerosis are more important in patients with tricuspid calcific stenosis than in those with a bicuspid aortic valve in whom mechanical wear and tear is more important.

We thank Dr Jan Poloniecki of Cardiological Sciences, S George's Hospital Medical School for expert statistical advice.

1 Edwards JE. On the etiology of calcific aortic stenosis. Circulation 1962;26:817-8.

2 Roberts WC. The structure of the aortic valve in clinically Roberts WC. The structure of the aortic valve in clinically
isolated aortic stenosis. An autopsy study of 162 patients isolated aortic stenosis. An autopsy study of 162 p

3 Roberts WC. The congenitally bicuspid aortic valve. A study of 85 autopsy cases. Am f Cardiol 1970;26:72-83. 
4 Davies MJ. Pathology of cardiac valves. London: Butterworth, 1980.

5 Subramanian R, Olson LJ, Edwards WD. Surgical pathol ogy of pure aortic stenosis a study of 374 cases. Mayo Clin Proc 1984;59:683-90.

6 Turri M, Thiene G, Bortolotti U, Milano A, Mazzucco A Gallucci V. Surgical pathology of aortic valve disease. A study based on 602 specimens. Eur $\mathcal{F}$ Cardiothorac Surg 1990;4:556-60.

7 Roberts WC. Anatomically isolated aortic valvular disease. The case against its being of rheumatic origin. $A m \mathcal{F} M e$ 1970;49:151-9.

8 Sadee AS, Becker AE, Verheul JA. The congenital bicuspid aortic valve with post-inflammatory disease-a neglected pathological diagnosis of clinical relevance. Eur Heart $f$ 1994;15:503-6.

Pomerance A. The pathogenesis of aortic stenosis and its relation to age. Br Heart f 1972;34:569-79.

10 Seltzer A. The changing aspects of aortic stenosis. $N$ Engl $f$ Med 1987;19:90.

11 Karalis DG, Wahl JM, Mintz GS, Chandrasekaran K Severe stenosis involving a congenitally bicuspid aortic valve in the tenth decade of life. Am $f$ Cardiol 1990 ; valve in

12 Edwards WD. Surgical pathology of the aortic valve. In: Contemporary issues in surgical pathology. Vol 12. New York: Longman (Churchill Livingstone), 1988:43-100.

13 Nistal JF, Garcia-Martinez V, Fernandez MD, Hurle A Hurle JM, Revuelta JM. Age-dependent dystrophic calcification of the aortic valve leaflets in normal subjects. $\mathcal{F}$ Heart Valve Dis 1994;3:37-40.

14 Lindroos M, Kupari M, Heikkila J, Tilvis R. Prevalence of aortic valve abnormalities in the elderly: an echocardiographic study of a random population sample. $7 \mathrm{Am} \mathrm{Coll}$ Cardiol 1993;21:1220-5.

15 Isner JM, Chokshi SK, DeFranco A, Braimen J, Slovenkai GA. Contrasting histoarchitecture of calcified leaflets from stenotic bicuspid versus stenotic tricuspid aortic valves. $\mathcal{A} \mathrm{Am}$ Coll Cardiol 1990;15:1104-8.

16 Vollebergh FEMG, Becker AE Minor congenital variations of cusp size in tricuspid aortic valves: possible link with isolated aortic stenosis. Br Heart $\mathcal{f} 1977 ; 39$ : 1006-11.

17 Otto CM, Kuusisto J, Reichenbach DD, Gown AM, $\mathrm{O}$ 'Brien $\mathrm{KD}$. Characterization of the early lesion of degenerative valvular aortic stenosis. Histological and immunohistochemical studies. Circulation 1994;90: 844-53.

18 Mautner GC, Mautner SL, Cannon RO III, Hunsberger SA, Roberts WC. Clinical factors useful in predicting aortic valve structure in patients $>40$ years of age with isolated valvular aortic stenosis. Am $\mathcal{F}$ Cardiol 1993;72: 194-8.

19 Mohler ER, Sheridan MJ, Nichols R, Harvey WP, Waller $B F$. Development and progression of aortic valve stenosis: atherosclerosis risk factors-a causal relationship? A clinical morphologic study. Clin Cardiol 1991;14:995-9.

20 Lindroos $M$, Kupari $M$, Valvanne J, Strandberg $T$, Heikkila J, Tilvis R. Factors associated with calcific aortic valve degeneration in the elderly. Eur Heart $\mathcal{f} 1994 ; 15$ : 865-70.

21 Strikberger SA, Schulman SP, Hutchins GM. Association of Paget's disease of bone with calcific aortic valve disof Paget's disease of bone with

22 Maher ER, Pazianas M, Curtis JR. Calcific aortic stenosis: a complication of chronic uraemia. Nephron 1987;47: 119-22.

23 Niederle B, Stefenelli T, Glogar D, Woloszczuk W, Roka $R$, Mayr H. Cardiac calcific deposits in patients with primary hyperparathyroidism: preliminary results of prospective echocardiographic study. Surgery 1990;108: $1052-7$.

24 Aronow WS, Schwartz KS, Koenigsberg H. Correlation of serum lipids, calcium and phosphorus, diabetes mellitus and history of systemic hypertension with presence or absence of calcified or thickened aortic cusps or root in elderly patients. Am $\mathcal{f}$ Cardiol 1987;59:998-9.

25 Ouchi Y, Akishita M, de Souza AC, Nakamura T, Orino $\mathrm{H}$. Age-related loss of bone mass and aortic/aortic valve calcification-revaluation of recommended dietary allowance of calcium in the elderly. Ann NY Acad Sci 1993;676:297-307. 